\title{
Los apósitos oclusivos en la ducha diaria ¿son capaces de reducir las infecciones del orificio de salida del catéter peritoneal?
}

Premio Baxter a la Investigación sobre Diálisis Peritoneal

Catalina Lima Comas - María José Alcantud García - Nuria Gámez Ráez - Adelaida Pastor Palenzuela Gonzalo Gómez Marqués - Antonio Morey Molina

Unidad de Diálisis Peritoneal. Servicio de Nefrología Hospital Universitario Son Dureta. Palma de Mallorca

\section{Resumen}

Las infecciones del orificio de salida del catéter peritoneal son causa de una elevada morbilidad en diálisis peritoneal, llegando a condicionar el mantenimiento de la técnica.

En anteriores estudios demostramos que el lavado del orificio de salida mediante agua previamente hervida, aplicada desde 1996, era capaz de disminuir las infecciones del orificio de salida por pseudomonas. En el año 2003, añadimos a la cura del orificio de salida con agua hervida, una protección durante la ducha diaria mediante apósitos oclusivos, que excluyera el contacto del orificio de salida con el agua doméstica.

Con este estudio estudio observacional en el que analizamos la densidad de incidencias en el orificio de salida queremos mostrar los resultados res-

\begin{tabular}{|c|}
\hline Correspondencia: \\
Catalina Lima Comas \\
Andrea Doria 55 \\
Hospital Universitario. Son Dureta \\
07014 Palma de Mallorca \\
capd@hsd.es \\
\hline
\end{tabular}

pecto a las infecciones, aplicando las dos medidas profilácticas en dos grupos de pacientes.

La combinación de lavado del orificio de salida con agua hervida, más los apósitos oclusivos se han mostrado efectivos en la profilaxis de las infecciones del orificio de salida.

\section{Palabras clave \\ DIÁLISIS PERITONEAL \\ CATÉTER \\ INFECCIÓN \\ APÓSITO}

Occlusive dressings in the shower - are they capable of reducing infections of the peritoneal catheter exit site?

\section{Abstract}

Infections of the peritoneal catheter exit site are the cause of high morbidity in peritoneal dialysis, and become a condition affecting maintenance of the technique. 
In previous studies we showed that washing the exit site with previously boiled water, applied since 1996, was capable of reducing exit-site infections caused by pseudomonas. In 2003, we added to the cleaning of the exit site with boiled water, protection whilst showering using occlusive dressings that excluded contact of the exit site with domestic water.

With this observational study in which we analyse the density of exit-site incidents, we wish to show the results concerning infections, applying the two prophylactic measures in two groups of patients.

The combination of washing the exit site with boiled water plus the occlusive dressings has been shown to be effective in preventing against infections of the exit site.

\section{Key words \\ PERITONEAL DIALYSIS \\ CATHETER \\ INFECTION \\ DRESSING}

\section{Introducción}

Las infecciones del orificio de salida del catéter peritoneal (IOS) son causa de una elevada morbilidad en diálisis peritoneal, llegando a condicionar el mantenimiento de la técnica. Más aún las provocada por Staphylococcus aureus (EA) y pseudomonas (PS), por sus particularidades invasivas y toxicogénicas ${ }^{1,2}$. Las pautas profilácticas contra el EA están bien establecidas con el uso de mupirocina, ácido fusídico y rifampicina. En el caso de las PS, bien adaptadas al medio acuoso, son vehiculizadas por el agua doméstica, donde incluso son capaces de reproducirse ${ }^{3,4}$, sin que la cloración sea capaz de evitarlo. En anteriores estudios demostramos que el lavado del orificio de salida (OS) mediante agua hervida (método que se aplicaba desde 1996), disminuía las IOS por PS, con un riesgo relativo de 6,15 veces menos de posibilidad de infección, siendo la densidad de incidencia de 0,66/paciente/año (p/a) en el grupo que no utilizó el agua hervida, frente a $0,10 / p / a$ en los que si la utilizaron ${ }^{5,6}$.
En otros estudios detectamos que en nuestro medio los pacientes en DP, incumplen las recomendaciones higiénico profilácticas, la ducha diaria y la cura del OS con agua hervida, siendo observado el cumplimiento de la ducha diaria en un $67 \%$, y la cura del OS en un $65 \%$.

\section{Objetivo}

Implementar una actitud profiláctica ante las IOS por PS, añadiendo a la cura del orificio de salida con agua hervida una protección durante la ducha diaria, mediante apósitos oclusivos, con el fin de excluir totalmente el contacto del OS con el agua doméstica.

\section{Material y métodos}

Estudio observacional en el que analizamos un total de 101 pacientes pertenecientes a nuestra Unidad de Diálisis Peritoneal, que se dividen en dos grupos, el grupo A compuesto de 55 pacientes (34 hombres), con una edad media 54,5 $\pm 14,4$, seguían el protocolo clásico de: ducha diaria sin retirada del apósito normal del OS e inmediatamente lavado del orificio con agua hervida. Valoramos la incidencia de IOS totales y de IOS por PS durante un período de 30 meses (de 1-2-2001 al 31-7-2003).

El grupo B, compuesto por 46 pacientes (25 hombres), con una edad media 54,5 $\pm 13,9$. Seguían el protocolo: colocar un apósito Oper-film ${ }^{\circledast}$ (membrana de poliuretano adhesiva, adaptable a la superficie cutánea, impermeable, no irritante y esterilizada por óxido de etileno), previamente a la ducha, al finalizar ésta, retirarlo y lavar con agua hervida el OS, junto con toda la zona cubierta con el apósito durante la ducha. El período del estudio fue también de 30 meses (del 1-8-2003 a 1-2-2006). Este segundo grupo se subdividió en dos:

- B no cumplidores (B no C), compuesto por los pacientes que incumplían la recomendación del uso de apósitos oclusivos en cinco o más duchas al mes, fueron un total de 15, 4 mujeres y 11 hombres, con una edad media de 59,4 $\pm 11,35$ años.

- B cumplidores (B C), que cumplimentaron adecuadamente el protocolo de apósitos oclusivos, fue- 
ron un total de 31 , compuesto por 15 hombres y 16 mujeres, con una edad media de $52,1 \pm 14,5$ años.

Estudiamos también nuestros resultados referidos a 1995 donde no se aplicaba ningún tipo de profilaxis. Denominamos Densidad de Incidencias (DI) a los episodios de infección del orificio por paciente y año.

A los pacientes del grupo B de les hacía las siguientes recomendaciones: la pared abdominal deberá permanecer rasurada y seca. Preparar el material para efectuar la cura del OS con agua hervida (protocolo habitual de nuestra unidad). Sin retirar el apósito de la cura anterior, cubrir el catéter y la línea de transferencia con una gasa, para evitar que el apósito se adhiera a ellos, lo que facilitará su retirada. Para colocar adecuadamente el apósito, se retira de un extremo la lámina protectora, se inicia el pegado por la zona lateral del abdomen, calculando que el centro del apósito oclusivo coincida con el centro del apósito normal, de forma progresiva se va colocando, retirando a la vez la lámina protectora, efectuando movimientos de arrastre y aplanamiento con la palma de la mano, procurando que no queden pliegues ni burbujas. El apósito, así colocado, cubrirá una zona de más de $3 \mathrm{~cm}$ alrededor del apósito normal (figuras 1 y 2).
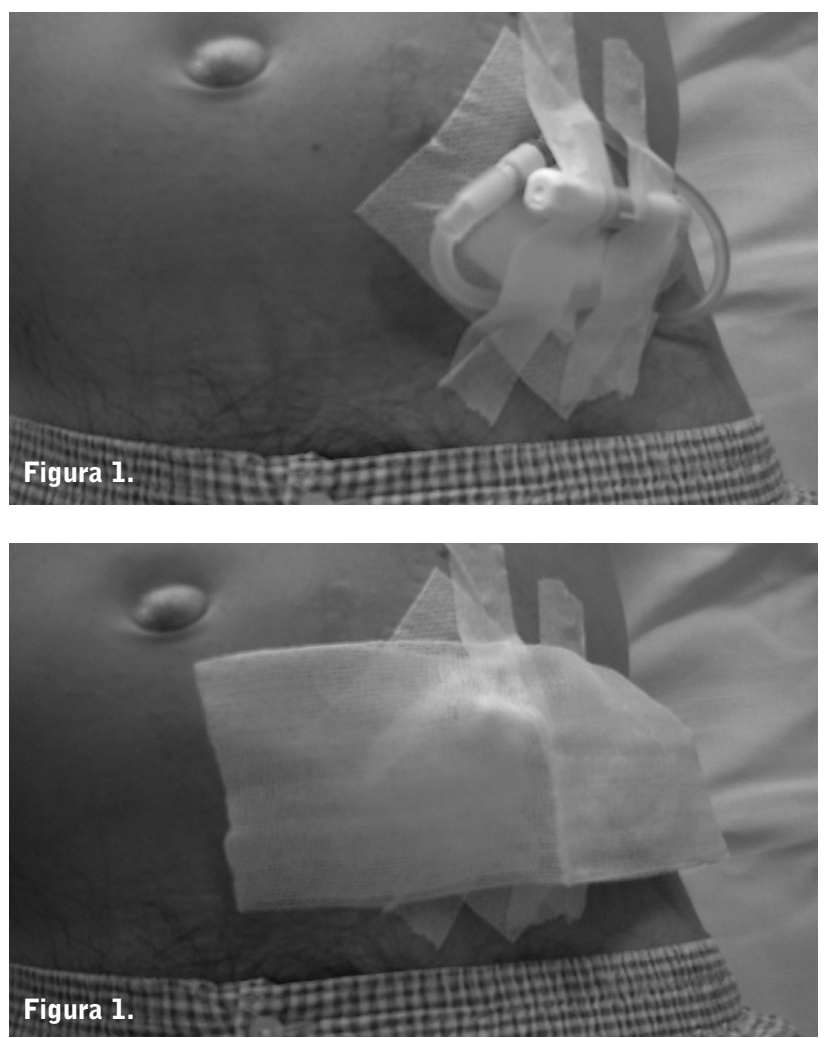
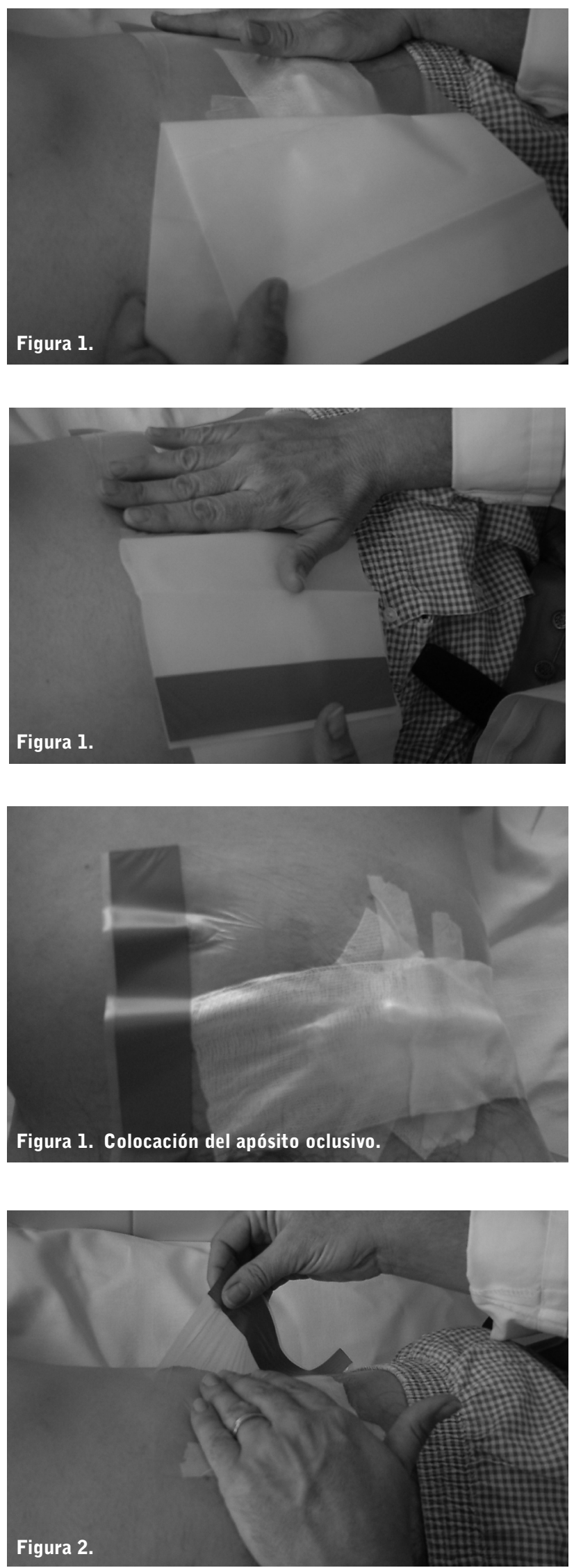

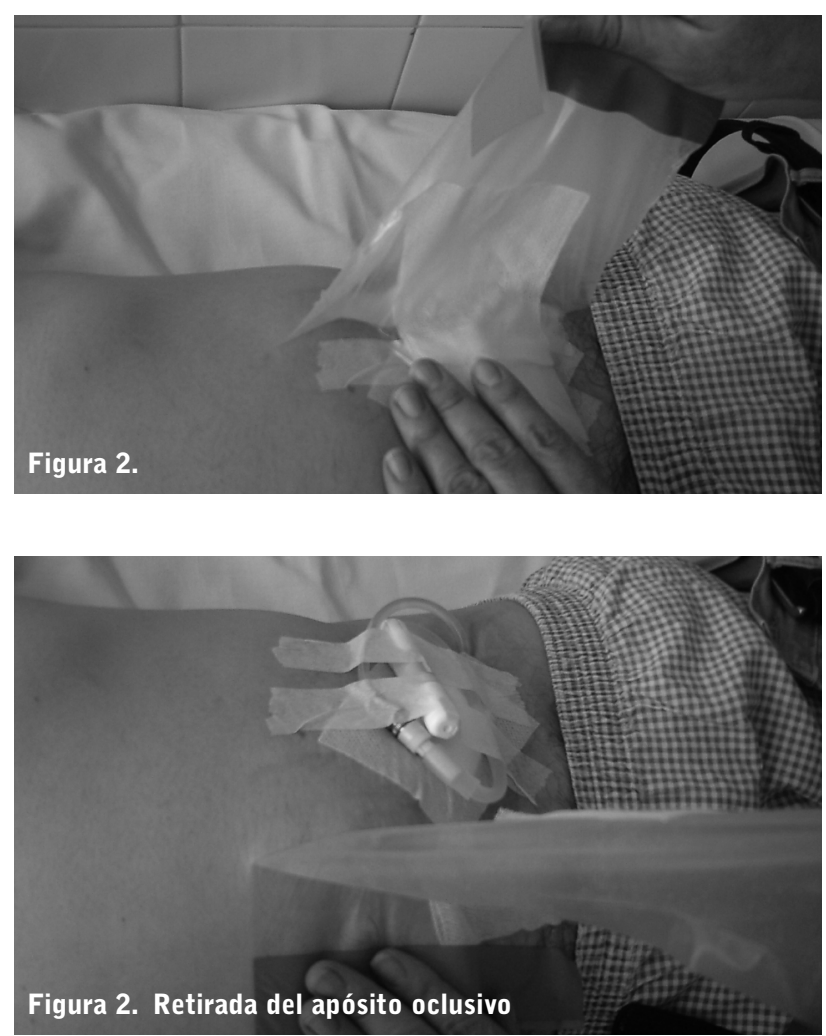

La ducha se efectuará con agua no excesivamente caliente, no más de cinco minutos, procurando no dirigir el chorro directamente sobre el apósito para evitar que se despegue. Una vez finalizada, se secará el tórax, parte superior del abdomen y extremidades superiores. Después de ponerse la mascarilla, se retiran los apósitos, quedando al descubierto el OS y se procederá al lavado de manos, y seguidamente al lavado del OS con agua hervida, además de toda la zona de pared abdominal que quedó cubierta por el apósito.

El método estadístico utilizado fue la comparación de medias mediante $t$ de Student.

\section{Resultados}

Todos los grupos resultaron homogéneos en cuando a la edad media, sexo y enfermedad renal primaria. De la comparación del grupo A y B no se han objetivado ninguna diferencia con respecto a las IOS totales y por PS (tabla 1). Cuando comparamos el grupo A frente al $B$ no $C$ de forma similar al anterior, tampoco obtenemos significación estadística (tabla 2). Los resultados de la comparación del grupo A con el B C, no arrojan unas diferencias significativas (tabla 3 ).

\begin{tabular}{|l|l|l|}
\hline & DI A & DI B \\
\hline IOS total & 1,02 & 0,94 \\
\hline IOS pseudomonas & 0,34 & 0,36 \\
\hline
\end{tabular}

Tabla 1. Densidad de Incidencias (DI) de los episodios de infección del orificio por paciente y año en ambos grupos.

\begin{tabular}{|l|l|l|}
\hline & DI A & DI B no C \\
\hline IOS total & 1,01 & 1,21 \\
\hline IOS pseudomonas & 0,34 & 0,57 \\
\hline
\end{tabular}

Tabla 2. Densidad de Incidencias (DI) de los episodios de infección del orificio por paciente y año del grupo A y del grupo B no cumplidores.

\begin{tabular}{|l|l|l|l|}
\hline & DI A & DI B C & p \\
\hline IOS total & 1.01 & 0,73 & 0,9 \\
\hline IOS pseudomonas & 0,34 & 0,20 & \\
\hline
\end{tabular}

Tabla 3. Densidad de Incidencias (DI) de los episodios de infección del orificio por paciente y año del grupo $\mathrm{A}$ y del grupo B cumplidores.

\begin{tabular}{|l|l|l|l|}
\hline & IOS total & DI & $p$ \\
\hline B C & 18 & 0,73 & \\
\hline B no C & 23 & 1,21 & 0,02 \\
\hline
\end{tabular}

Tabla 4. Densidad de Incidencias (DI) de los episodios de infección total del orificio por paciente y año del grupo B (cumplidores y no cumplidores).

\begin{tabular}{|l|c|c|c|}
\hline & IOS pseudomonas & DI & $p$ \\
\hline B C & 5 & 0,20 & \multirow{2}{*}{0,01} \\
\hline B no C & 11 & 0,57 & \\
\hline
\end{tabular}

Tabla 5. Densidad de Incidencias (DI) de los episodios de infección por pseudomonas del orificio por paciente y año del grupo B (cumplidores y no cumplidores).

\begin{tabular}{|c|c|c|c|c|}
\hline & $N^{0}$ pacientes & IOS pseudomonas & DI & $p$ \\
\hline 1995 & 22 & 11 & 0,68 & \multirow{2}{*}{0,03} \\
\hline 2005 & 29 & 3 & 0,15 & \\
\hline
\end{tabular}

Tabla 6. Densidad de Incidencias (DI) de los episodios de infección por pseudomonas del orificio por paciente y año entre los años 1995 (sin protocolo) y 2005 (con protocolo). 
De la valoración de los grupos B C y B no C se derivan diferencias importantes, tanto en el número de infecciones del OS totales como por pseudomonas (tabla 4 y 5 ).

Los resultados obtenidos comparando los años 1995 (que no se aplicaba ninguna medida profiláctica) y 2005 (que se usó como profilaxis el lavado del OS con agua hirviendo y el apósito oclusivo para la ducha) en cuanto a las infecciones del OS por pseudomonas nos dan unas diferencias importantes (tabla 6).

\section{Discusión}

La implantación del protocolo de limpieza del OS con agua hervida, que proporciona la exclusión del contacto del agua de la ducha con el OS, y que se lleva a cabo en nuestra unidad desde 1996, condujo a una drástica reducción de las IOS totales y por PS, con altos grados de significación estadística y una disminución del riesgo relativo de hasta 6,15 veces para las IOS por PS y de 3 para las IOS a cualquier germen ${ }^{6}$.

La utilización del agua hervida en este estudio es utilizada en ambos grupos, por lo que la reducción de las IOS no es significativa entre el grupo $A$ y el grupo $B$ total; esto nos lleva a deducir que el agua hervida tiene una capacidad profiláctica mucho más elevada que la utilización de los apósitos, y en esta ocasión la primera medida profiláctica está plenamente implantada en ambos grupos.

Comparando el grupo A con el grupo B no C, es decir, el que no cumple el protocolo de los apósitos oclusivos, tampoco obtiene significación estadística, probablemente a la vista del mayor número de infecciones del grupo $B$ no $C$ podríamos sospechar que incumplen también con el protocolo de cura de la ducha diaria y la cura con agua hervida, ya que sólo hemos valorado el protocolo del apósito y no hemos medido en este estudio el nivel de cumplimiento de la ducha y la cura con agua hervida.

$Y$ en cuanto a los resultados obtenidos entre el grupo B C y B no $C$ si que encontramos una diferencia significativa ya que los pacientes que cumplen el protocolo, tienen menos IOS totales que los que no cumplen. Por otra parte, se demuestra también una clara disminución de las infecciones por PS entre cumplidores y no cumplidores.
Finalmente, comparando el número de IOS por PS del año 1995, donde no utilizábamos el agua hervida ni los apósitos oclusivos, y el número de IOS por PS en el año 2005, en el que si utilizábamos el agua hervida junto con los apósitos oclusivos, podemos observar una diferencia significativa, lo que nos lleva a demostrar que el agua hervida junto con los apósitos oclusivos disminuyen las IOS por pseudomonas.

\section{Conclusiones}

Consideramos que el agua hervida es la principal arma profiláctica frente a las infecciones del orificio de salida del catéter y que la combinación del lavado más los apósitos oclusivos son efectivos para la profilaxis de las IOS en general y para las infecciones por pseudomonas. La aplicación de ambas medidas profilácticas nos ha permitido reducir notablemente el riesgo de infección del orificio.

\section{Bibliografía}

1. Montenegro J. Prevención y tratamiento de la infección del orificio del catéter peritoneal. Nefrología. 1999; 19:502-507.

2. Morey A, Lima C, Munar MA y Martínez JG. Estrategias de prevención de la infección del orificio de salida del catéter peritoneal. Nefrología. 2000; 20 (3):290.

3. Sheng-jeng P, Chewei-Shiun Y, Shyang-Hwa F. The Clinical experience and natural course of peritoneal catheter exit site infection amoung continuous ambulatory peritoneal dialysis patients. Dialysi Transplantation.1998; 27:71-78.

4. Chi Yuen L, Wai Ling C, Kit Mui W, Suk Yi N, Ling L, Mui Fun C, Suk Wai C, Wai Kei L. Pseudomonas exit-site infections in CAPD patients: evolution and outcome of treatment. Perit Dial Int. 1998; 18: 637-640.

5. Lima C, Matas B, Alcantud MJ, Pastor A, Munar MA, Morey A. Encuesta Valoración del cumplimiento de las normas higiénico-profilácticas en diálisis peritoneal. IV Reunión Nacional de Diálisis Peritoneal. 2003. Barcelona.

6. Morey A, Munar MA, Lima C, Matas B, Losada P, De la Prada F, Martínez JG, Gómez G, Alarcón A. Profilaxis frente a las infecciones por pseudomonas del orificio de salida del catéter de diálisis peritoneal. XXIV Congreso Nacional de la Sociedad Española de Diálisis y Trasplante. 2002. Teruel 